\title{
Isothermal-sweep theorems for ultracold quantum gases in a canonical ensemble
}

\author{
M. Iskin \\ Department of Physics, Koç University, Rumelifeneri Yolu, TR-34450 Sariyer, Istanbul, Turkey \\ (Received 20 September 2010; revised manuscript received 10 February 2011; published 16 March 2011)
}

\begin{abstract}
After deriving the isothermal Hellmann-Feynman theorem (IHFT) that is suitable for mixed states in a canonical ensemble, we use this theorem to obtain the isothermal magnetic-field sweep theorems for the free, average, and trapping energies and for the entropy, specific heat, pressure, and atomic compressibility of strongly correlated ultracold quantum gases. In particular, we apply the sweep theorems to two-component Fermi gases in the weakly interacting Bardeen-Cooper-Schrieffer and Bose-Einstein condensate limits, showing that the temperature dependence of the contact parameter can be determined by varying either the entropy or specific heat with respect to the scattering length. We also use the IHFT to obtain the virial theorem in a canonical ensemble and discuss its implications for quantum gases.
\end{abstract}

DOI: 10.1103/PhysRevA.83.033613

PACS number(s): 03.75.Ss, 03.75.Hh

\section{INTRODUCTION}

Dilute samples of ultracold quantum gases are well described by zero-range interactions, a consequence of which is the existence of some universal relations that govern the behavior of these systems [1]. The first examples of such relations were originally derived by solving the many-body Schrödinger equation [2-4], and they relate energy and pressure of the system to a single parameter that comes from the short-range physics. What makes these relations remarkable is that they hold for any finite-energy state of the system; it does not matter whether the system is few- or manybody, superfluid or normal, weakly or strongly interacting, in equilibrium or nonequilibrium, or at zero or finite temperature. These universal relations have more recently been rederived using many other approaches, including the quantum-fieldtheoretical techniques [1,5-9], and have also been verified via numerical few-body calculations [10].

There is one common element in these universal relations: they all involve a single parameter called the contact. In the case of two-component Fermi gases, the contact parameter is given by the coefficient of the $1 / k^{4}$ tail of the momentum distribution, and therefore, it measures the number of fermion pairs with small separations. It has been found that the contact parameter also appears in many other short-range (high-momentum) or short-time (high-frequency) properties of the system [11-16]. This parameter has recently been measured in an ultracold ${ }^{40} \mathrm{~K}$ gas via the measurements of the high-momentum tail of the momentum distribution and of the high-frequency tail of the radio-frequency signal [17] and has also been measured in an ultracold ${ }^{6} \mathrm{Li}$ gas via the measurements of the static structure factor [18]. The measured temperature and scattering length dependence of the contact parameter compare well with the theoretical predictions $[19,20]$.

In particular, the so-called adiabatic-sweep theorem for the energy [3], which has recently been experimentally verified [17], relates the contact to the change in total energy of the system when the atom-atom scattering length $a$ is changed adiabatically, i.e., with zero heat transfer. In atomic systems, the value of $a$ can be tuned at will from very large and negative to very large and positive values because of the presence of magnetically induced Feshbach resonances, and an adiabatic (constant entropy) sweep is accomplished by changing the bias magnetic field over a time scale longer than the relaxation time [17].

Motivated by these earlier works, here we generalize a number of these universal relations to finite temperatures, and the rest of the paper is organized as follows. In Sec. II, we derive the isothermal Hellmann-Feynman theorem (IHFT) that is suitable for mixed states in a canonical ensemble. Then, we use this theorem in Sec. III to obtain the isothermal (constant temperature) sweep theorems (IST) for the free, average, and trapping energies and for the entropy, specific heat, pressure, and atomic compressibility of strongly correlated ultracold Fermi gases. We also use the IHFT to derive the virial theorem in a canonical ensemble in Sec. IV. A brief summary of our conclusions is given in Sec. V.

\section{HELLMANN-FEYNMAN THEOREMS}

For our purpose, we need to derive the Hellmann-Feynman theorem (HFT) suitable for a canonical ensemble of a fixed number $N$ of identical particles that are in thermal equilibrium with a heat reservoir, which is discussed next.

\section{A. HFT for pure states}

For any stationary normalized eigenvector $\left|\psi_{n}(\lambda)\right\rangle$ with the corresponding eigenvalue $E_{n}(\lambda)$ of a Hamiltonian $H(\lambda)$, i.e., $\left\langle\psi_{n} \mid \psi_{n}\right\rangle=1$ and $E_{n}=\left\langle\psi_{n}|H| \psi_{n}\right\rangle$, the usual HFT states that

$$
\frac{\partial E_{n}}{\partial \lambda}=\left\langle\psi_{n}\left|\frac{\partial H}{\partial \lambda}\right| \psi_{n}\right\rangle,
$$

where $\lambda$ is an arbitrary real parameter, explicitly appearing in $H$. This well-known theorem has proved to be very useful in many fields ranging from quantum chemistry, quantum statistics, and many-body physics to molecular physics, with many applications.

In particular, the HFT has recently been used to derive a number of exact relations for strongly correlated systems with short-range interactions, in the context of ultracold quantum gases [5,6,21]. Since this theorem applies only for pure states, these exact relations strictly hold at zero temperature for any change or equivalently at finite temperatures for an adiabatic change. In order to generalize these relations to 
finite temperatures, here we derive the IHFT for a canonical ensemble of a fixed number of identical particles that are in thermal equilibrium with a heat reservoir.

\section{B. IHFT for mixed states in a canonical ensemble}

For this purpose, we assume the temperature $T$ is fixed and the Hamiltonian $H$ is a function of some arbitrary real parameter $\lambda$. There are two complimentary ways to obtain the isothermal HFT in a canonical ensemble.

The simplest way follows from the definition of the statistical Helmholtz free energy $F$, i.e., $F=-T \ln Z$ in a unit of $k_{B}=1$ here and throughout, where $Z=\operatorname{Tr}\left(e^{-H / T}\right)$ is the canonical partition function of the system. Then, it is easy to show that (see also [22,23])

$$
\frac{\partial F}{\partial \lambda}=\left\langle\frac{\partial H}{\partial \lambda}\right\rangle
$$

where the ensemble average of any operator is defined by $\langle A\rangle=\operatorname{Tr}(\rho A)$ and $\rho=e^{-H / T} / Z$ is the density operator in the canonical ensemble. We recall that the derivatives with respect to $\lambda$ are evaluated at fixed $T$ here and throughout. In addition, using the definitions of the thermodynamic free energy, average energy, and entropy, i.e., $F=E-T S, E=$ $\langle H\rangle$, and $S=-\operatorname{Tr}(\rho \ln \rho)=-\partial F / \partial T$, respectively, Eq. (2) can be written as

$$
\frac{\partial E}{\partial \lambda}=\left(1-T \frac{\partial}{\partial T}\right)\left\langle\frac{\partial H}{\partial \lambda}\right\rangle .
$$

This is the isothermal HFT for mixed states in a canonical ensemble, and it will play a central role in the rest of this paper.

An alternative way of deriving Eq. (3) is as follows. We start directly from the definition of $E$, i.e. $E=\operatorname{Tr}(\rho H)$, and evaluate the derivative to obtain

$$
\frac{\partial E}{\partial \lambda}=\left\langle\left(1+\frac{E-H}{T}\right) \frac{\partial H}{\partial \lambda}\right\rangle .
$$

In the intermediate steps, we used $\operatorname{Tr}\left(\partial e^{-H / T} / \partial \lambda\right)=$ $-(1 / T) \operatorname{Tr}\left(e^{-H / T} \partial H / \partial \lambda\right)$ and $\operatorname{Tr}\left(H \partial e^{-H / T} / \partial \lambda\right)=-(1 / T)$ $\operatorname{Tr}\left(H e^{-H / T} \partial H / \partial \lambda\right)$, both of which can be derived by writing $\operatorname{Tr}(A)=\sum_{n}\left\langle\psi_{n}|A| \psi_{n}\right\rangle$, where $\left|\psi_{n}\right\rangle$ is the normalized eigenvector and $E_{n}$ is the corresponding eigenvalue of $H$. In addition, we used $\partial e^{-E_{n} / T} / \partial \lambda=\left\langle\psi_{n}\left|\partial e^{-H / T} / \partial \lambda\right| \psi_{n}\right\rangle$, which can be obtained from the usual HFT given in Eq. (1). For fixed $T$, starting from the definition $\langle A\rangle=\operatorname{Tr}(\rho A)$, it is easy to show for the observables that commute with $H$, i.e., $[H, A]=0$, that $\partial\langle A\rangle / \partial T=\langle(H-E) A\rangle / T^{2}$. Therefore, Eqs. (3) and (4) are equivalent.

Having derived the IHFT for a canonical ensemble, next we use this theorem to obtain a number of exact relations for strongly correlated ultracold quantum gases, which is the main purpose of this paper.

\section{ISOTHERMAL-SWEEP THEOREMS}

In this section, we use the IHFT given in Eq. (3) to derive the IST for the free, average, and trapping energies and for the entropy, specific heat, pressure, and atomic compressibility in a canonical ensemble.

\section{A. IST for free, average, and trapping energies}

As a first application of the IHFT, we consider the Hamiltonian that describes two-component Fermi gases in an external potential $U_{\sigma}(\mathbf{r})$ :

$$
\begin{aligned}
H= & \sum_{\sigma} \int d^{3} \mathbf{r} \psi_{\sigma}^{\dagger}(\mathbf{r})\left[-\frac{\hbar^{2} \nabla^{2}}{2 m_{\sigma}}+U_{\sigma}(\mathbf{r})\right] \psi_{\sigma}(\mathbf{r}) \\
& -g \int d^{3} \mathbf{r} \psi_{\uparrow}^{\dagger}(\mathbf{r}) \psi_{\downarrow}^{\dagger}(\mathbf{r}) \psi_{\downarrow}(\mathbf{r}) \psi_{\uparrow}(\mathbf{r}),
\end{aligned}
$$

where $\psi_{\sigma}^{\dagger}(\mathbf{r})$ creates a pseudospin- $\sigma$ fermion with mass $m_{\sigma}$ and $g \geqslant 0$ is the strength of the short-range interaction. In the following discussions, the numbers of $\uparrow$ and $\downarrow$ fermions need not be equal. As usual, the theoretical parameter $g$ can be written in terms of the experimentally more relevant scattering length $a$ via $1 / g=-M V /\left(4 \pi \hbar^{2} a\right)+\left(M / \hbar^{2}\right) \sum_{k}\left(1 / k^{2}\right)$, where $M=2 m_{\uparrow} m_{\downarrow} /\left(m_{\uparrow}+m_{\downarrow}\right)$ is twice the reduced mass of $\uparrow$ and $\downarrow$ fermions and $V$ is the volume. This equation gives $g=$ $-4 \pi^{2} \hbar^{2} a /\left(M V \pi-2 M V a k_{c}\right)$, where $k_{c}$ is the momentumspace cutoff used to evaluate the ultraviolet divergent sum over $\mathbf{k}$.

Following the recent work of Ref. [5], the contact parameter $C$ can be defined as

$$
\begin{aligned}
\left\langle\frac{\partial H}{\partial a}\right\rangle & =\frac{M V g^{2}}{4 \pi \hbar^{2} a^{2}} \int d^{3} \mathbf{r}\left\langle\psi_{\uparrow}^{\dagger}(\mathbf{r}) \psi_{\downarrow}^{\dagger}(\mathbf{r}) \psi_{\downarrow}(\mathbf{r}) \psi_{\uparrow}(\mathbf{r})\right\rangle, \\
& =\frac{\hbar^{2} C}{4 \pi M a^{2}}
\end{aligned}
$$

where we used $d g / d a=-M V g^{2} /\left(4 \pi \hbar^{2} a^{2}\right)$. Note that $C$ is an extensive quantity that depends on both $a$ and $T$. This definition guarantees [5] that the average energy $E$ of the system is of the desired form [2,8],

$$
E-\sum_{\sigma}\left\langle U_{\sigma}\right\rangle=\sum_{\sigma, \mathbf{k}} \frac{\hbar^{2} k^{2}}{2 m_{\sigma}}\left[n_{\sigma}(\mathbf{k})-\frac{C}{k^{4}}\right]+\frac{\hbar^{2} C}{4 \pi M a},
$$

where $n_{\sigma}(\mathbf{k})$ is the momentum distribution of $\sigma$ fermions. As emphasized in [2], this relation holds for any finite-energy state of the system; it does not matter whether the system is few- or many-body, superfluid or normal, weakly or strongly interacting, in equilibrium or nonequilibrium, or at zero or finite temperature. Combining Eq. (7) with the IHFT given in Eqs. (2) and (3), we obtain

$$
\begin{gathered}
\frac{\partial F}{\partial a}=\frac{\hbar^{2} C}{4 \pi M a^{2}}, \\
\frac{\partial E}{\partial a}=\frac{\hbar^{2}}{4 \pi M a^{2}}\left(C-T \frac{\partial C}{\partial T}\right) .
\end{gathered}
$$

Here the derivatives with respect to $a$ are evaluated at fixed $T$, and therefore, Eqs. (9) and (10) correspond to the IST for the free and average energies, respectively. Compared to the zero-temperature expression for any change or, equivalently, the finite-temperature expression for an adiabatic change, i.e., the adiabatic-sweep theorem for the energy [1,3], the main difference in Eq. (10) is an extra $T \partial C / \partial T$ term. Similarly, we can also calculate $\partial F / \partial k_{c}=\hbar^{2} C /(2 \pi M)$ and $\partial E / \partial k_{c}=\left[\hbar^{2} /(2 \pi M)\right](C-T \partial C / \partial T)$, where we used $d g / d k_{c}=-M V g^{2} /\left(2 \pi^{2} \hbar^{2}\right)$. 
For homogenous systems (no external potential), the $T$ dependence of $C$ has been recently calculated in the low-, intermediate-, and high- $T$ regimes as [19]

$$
\begin{gathered}
C=C_{0}+\alpha_{1} T^{4}\left(T \ll T_{c}<T_{F}\right), \\
C=C_{0}+\alpha_{2} T^{2}\left(T_{c}<T \ll T_{F}\right), \\
C=\frac{\alpha_{3}}{T}\left(T \gg\left\{T_{F}, T_{a}\right\}\right),
\end{gathered}
$$

where, up to the leading orders in $a, C_{0}=4 \pi^{2} n^{2} a^{2}, \alpha_{1}=$ $9 \sqrt{3} \pi^{6} n^{2} a^{2} /\left(40 T_{F}^{4}\right), \quad$ and $\quad \alpha_{2}=-4(7 \ln 2-1) \pi^{3} k_{F} n^{2} a^{3} /$ $\left(5 T_{F}^{2}\right)$ in the Bardeen-Cooper-Schrieffer (BCS) limit; $C_{0}=$ $2 \pi n / a$ and $\alpha_{1}=2 \pi^{6} \sqrt{\pi k_{F} / a}\left(\partial a_{m} / \partial a\right) n / T_{F}^{4}$ in the BoseEinstein condensate (BEC) limit; and $\alpha_{3}=8 \pi^{2} n^{2} / M$ for all couplings. Here $k_{F}$ is the Fermi momentum, $T_{c}$ is the critical temperature for superfluidity, $T_{F}=\hbar^{2} k_{F}^{2} /(2 M)$ is the Fermi temperature, $T_{a}=\hbar^{2} /\left(M a^{2}\right)$ is the binding energy of two fermions, $n=N / V=k_{F}^{3} /\left(3 \pi^{2}\right)$ is the density of fermions, $a_{m}$ is the dimer-dimer scattering length between Cooper molecules (e.g., $a_{m}=0.6 a$ when $m_{\uparrow}=m_{\downarrow}$ ), and the weakly interacting BCS and BEC limits are characterized by $k_{F} a \rightarrow 0^{-}$and $k_{F} a \rightarrow 0^{+}$, respectively. Note that our definition of $C$ is larger by a factor of $4 \pi^{2}$ compared to the definition of Ref. [19]. Note also that the expression for intermediate- $T$ regime, i.e., Eq. (12), is valid only in the BCS limit and that $C$ has a maximum at a particular $T$ since $C_{0}, \alpha_{1}, \alpha_{2}$, and $\alpha_{3}$ are all positive constants with respect to $T$. Using Eqs. (11)-(13) in Eq. (10), we find $\partial E / \partial a=\hbar^{2}$ $\left(C_{0}-3 \alpha_{1} T^{4}\right) /\left(4 \pi M a^{2}\right), \partial E / \partial a=\hbar^{2}\left(C_{0}-\alpha_{2} T^{2}\right) /\left(4 \pi M a^{2}\right)$, and $\partial E / \partial a=\hbar^{2} C /\left(2 \pi M a^{2}\right)$ for the low-, intermediate-, and high- $T$ regimes, respectively.

Note also that taking the derivative of the virial theorem given in Eq. (29) with respect to $a$ and using the IST for the energy given in Eq. (10), we obtain

$$
\frac{\partial E_{t r}}{\partial a}=\frac{\hbar^{2}}{16 \pi M a^{2}}\left(C+a \frac{\partial C}{\partial a}-2 T \frac{\partial C}{\partial T}\right),
$$

which corresponds to the IST for the trapping energy. Here

$$
E_{t r}=\frac{1}{2}\left\langle U+\frac{1}{2} \sum_{i=1}^{N} \mathbf{r}_{\mathbf{i}} \cdot \nabla_{\mathbf{r}_{\mathbf{i}}} U\right\rangle
$$

is the effective trapping energy, which reduces to the trapping energy $\langle U\rangle$ in the case of harmonic trapping potentials.

\section{B. IST for entropy and specific heat}

The IHFT can also be used to find other thermodynamic relations. For instance, we obtain an entropy relation by taking the derivative of $S=(E+F) / T$ with respect to $\lambda$ and using Eqs. (2) and (3), leading to

$$
\frac{\partial S}{\partial \lambda}=-\frac{\partial}{\partial T}\left\langle\frac{\partial H}{\partial \lambda}\right\rangle \text {. }
$$

In addition, we obtain a specific-heat relation (at constant volume) from $C_{V}=\partial E / \partial T=T \partial S / \partial T$, leading to

$$
\frac{\partial C_{V}}{\partial \lambda}=-T \frac{\partial^{2}}{\partial T^{2}}\left\langle\frac{\partial H}{\partial \lambda}\right\rangle .
$$

In Eqs. (16) and (17) the derivatives with respect to $\lambda$ are evaluated at fixed $T$. We hope that these relations could be tested with thermodynamic measurements in strongly interacting Fermi gases [24].

For ultracold quantum gases described by the Hamiltonian given in Eq. (5), we can use Eq. (7) in Eqs. (16) and (17), leading to

$$
\begin{aligned}
\frac{\partial S}{\partial a} & =-\frac{\hbar^{2}}{4 \pi M a^{2}} \frac{\partial C}{\partial T}, \\
\frac{\partial C_{V}}{\partial a} & =-\frac{\hbar^{2} T}{4 \pi M a^{2}} \frac{\partial^{2} C}{\partial T^{2}} .
\end{aligned}
$$

Here the derivatives with respect to $a$ are evaluated at fixed $T$, and therefore, Eqs. (18) and (19) correspond to the IST for the entropy and specific heat, respectively. Equation (18) was first derived in Ref. [19]. Using Eqs. (11)-(13) in Eqs. (18) and (19), we find $\partial S / \partial a=-\alpha_{1} T^{3} /\left(\pi M a^{2}\right)$ and $\partial C_{V} / \partial a=$ $-3 \alpha_{1} T^{3} /\left(\pi M a^{2}\right)$ for the low $T, \partial S / \partial a=-\alpha_{2} T /\left(2 \pi M a^{2}\right)$ and $\partial C_{V} / \partial a=-\alpha_{2} T /\left(2 \pi M a^{2}\right)$ for the intermediate $T$, and $\partial S / \partial a=C /\left(4 \pi M a^{2} T\right)$ and $\partial C_{V} / \partial a=-C /\left(2 \pi M a^{2} T\right)$ for the high- $T$ regimes. Therefore, since $\alpha_{1}$ and $\alpha_{2}$ are positive constants, while $\partial S / \partial a$ is negative for low $T$, it becomes positive at high $T$, indicating that $\partial S / \partial a$ vanishes at a particular temperature above $T_{F}$. Note also that $\partial S / \partial a \propto$ $\partial C_{V} / \partial a$ in these regimes and that the $T$ dependence of $C$ could be determined by varying either $S$ or $C_{V}$ with respect to $a$.

\section{IST for pressure}

For the Hamiltonian given in Eq. (5) but in the absence of the potential term, i.e., a homogenous system where $U(\mathbf{r})=0$, the IHFT can be used to derive the IST for the pressure.

In general, via a dimensional analysis, the free energy $F$ can be written as $F\left(\eta_{1}, \ldots, \eta_{r}\right)=\left(\hbar^{2} \lambda^{2} / M\right) f\left(\lambda \eta_{1}, \ldots, \lambda \eta_{r}\right)$, where $\lambda$ has the dimension of the inverse of a length, $f$ is a dimensionless function of its parameters, and $\eta_{j}$ labels $r$ parameters (all with the dimension of a length) that $F$ may depend on for a given $H$. For homogenous ultracold quantum gases, since $F$ is a function of $T, V, N_{\sigma}$, and $a$, dimensional analysis [6] requires that $F$ must satisfy

$$
2 F=-\sum_{q=1}^{r} \eta_{q} \frac{\partial F}{\partial \eta_{q}}=-a \frac{\partial F}{\partial a}+2 T \frac{\partial F}{\partial T}-3 V \frac{\partial F}{\partial V} .
$$

Using $F=E-T S, S=-\partial F / \partial T, P=-\partial F / \partial V$, and the IST for the free energy given in Eq. (9), we obtain

$$
P=\frac{2 E}{3 V}+\frac{\hbar^{2} C}{12 \pi M a V} \text {. }
$$

Therefore, the universal pressure relation in a canonical ensemble is of the same form as the zero-temperature one $[1,3]$.

Taking the derivative of Eq. (21) with respect to $a$ and using the IST for the energy given in Eq. (10), we obtain

$$
\frac{\partial P}{\partial a}=\frac{\hbar^{2}}{12 \pi M a^{2} V}\left(C+a \frac{\partial C}{\partial a}-2 T \frac{\partial C}{\partial T}\right),
$$

which corresponds to the IST for the pressure. We recall that Eq. (22) is derived for a homogenous system, and therefore, it is incorrect to compare it with Eq. (14) and conclude that $3 V \partial P / \partial a=4 \partial E_{t r} / \partial a$ for a trapped system. Using Eqs. (11)-(13) in Eq. (22), we find 
$\partial P / \partial a=\hbar^{2}\left(3 C_{0}-5 \alpha_{1} T^{4}\right) /\left(12 \pi M a^{2} V\right)$ in the BCS and $\partial P / \partial a=-5 \hbar^{2} \alpha_{1} T^{4} /\left(8 \pi M a^{2} V\right)$ in the BEC limit for the low- $T$ regime, $\partial P / \partial a=\hbar^{2} C_{0} /\left(4 \pi M a^{2} V\right)$ in the BCS limit for the intermediate- $T$ regime, and $\partial P / \partial a=\hbar^{2} C /\left(4 \pi M a^{2} V\right)$ for the high- $T$ regime. Therefore, since $\alpha_{1}$ is a positive constant, while $\partial P / \partial a$ is positive in the BCS limit, it becomes negative in the BEC limit in the low- $T$ regime, indicating that $\partial P / \partial a$ vanishes at a particular scattering length around unitarity.

\section{IST for atomic compressibility}

The isothermal atomic compressibility is defined as $\kappa_{T}=$ $-V^{-1} \partial V / \partial P$, and it can be obtained by taking the derivative of Eq. (21) with respect to $V$ at constant $T$. Using Eq. (9) to relate $\partial C / \partial V$ to $P, P=-\partial F / \partial V$, and $E=F+T S$ together with the Maxwell relation $\partial S /\left.\partial V\right|_{T}=\partial P /\left.\partial T\right|_{V}$, we obtain

$$
\frac{3}{\kappa_{T}}=5 P+a \frac{\partial P}{\partial a}-2 T \frac{\partial P}{\partial T} .
$$

This equation also follows from a dimensional analysis of the pressure $P$, which is a function of $T, V, N_{\sigma}$, and $a$, similar to the analysis that led to Eq. (20). The $\partial P / \partial a$ term is given by Eq. (22), and the $\partial P / \partial T$ term can be obtained by taking the derivative of Eq. (21) with respect to $T$, leading to

$$
C_{V}=\frac{3 V}{2} \frac{\partial P}{\partial T}-\frac{\hbar^{2}}{8 \pi M a^{2}} \frac{\partial C}{\partial T},
$$

where $C_{V}=\partial E / \partial T$ is the specific heat at constant $V$. Note that this equation relates the specific heat to the pressure and contact parameter. Using Eqs. (22) and (19) in Eq. (23), we obtain

$$
\frac{3}{\kappa_{T}}=5 P-\frac{4 T}{3 V} C_{V}+\frac{\hbar^{2}}{12 \pi M a V}\left(C+a \frac{\partial C}{\partial a}-4 T \frac{\partial C}{\partial T}\right),
$$

which relates the isothermal atomic compressibility to the pressure, specific heat, and contact parameter. When $C=0$, Eq. (25) is satisfied for the ideal Fermi gases. The IST for the atomic compressibility can be easily obtained by taking the derivative of Eq. (25) with respect to $a$ and using Eqs. (22) and (19).

Note that since the compressibility is related to the density fluctuations via the fluctuation-dissipation theorem, $\kappa_{T}=$ $V T^{-1}\left(\left\langle\hat{N}^{2}\right\rangle-\langle\hat{N}\rangle^{2}\right) /\langle\hat{N}\rangle^{2}$, where $\hat{N}$ is the density operator, it can be used to extract some thermodynamic information in atomic systems. Although this was proposed in as early as 2005 [25], it has recently been possible to extract this information for two-component Fermi gases by measuring the density fluctuations and atomic compressibility [26,27]. Combining Eq. (25) with the fluctuation-dissipation theorem provides yet another universal relation that can be verified with atomic systems. Note also that the isoentropic (or adiabatic) compressibility $\kappa_{S}$ is related to $\kappa_{T}$ via $\kappa_{S} / \kappa_{T}=C_{V} / C_{P}$, where $C_{P}$ is the specific heat at constant $P$. Since the specific heats are also related to each other via $C_{P}=C_{V}+T V \kappa_{T}(\partial P / \partial T)^{2}$, using Eq. (24), we obtain

$$
\frac{1}{\kappa_{S}}=\frac{1}{\kappa_{T}}+\frac{T V}{C_{V}}\left(\frac{2 C_{V}}{3 V}+\frac{\hbar^{2}}{12 \pi M a V} \frac{\partial C}{\partial T}\right)^{2},
$$

which relates $\kappa_{S}$ to $\kappa_{T}, C_{V}$, and $C$. In atomic systems, it is easier to measure $\kappa_{S}$ than $\kappa_{T}$, and Eq. (26) can be used to extract the temperature dependence of $C$, given that $\kappa_{T}$ can be extracted from the fluctuation-dissipation theorem [26,27].

Having derived the IST for the free, average, and trapping energies and for the entropy, specific heat, pressure, and atomic compressibility in a canonical ensemble, next we derive the virial theorem.

\section{VIRIAL THEOREM FOR TRAPPED SYSTEMS}

In this section, we use the IHFT given in Eq. (3) to derive the virial theorem $[4,21,22,24]$ in a canonical ensemble. This can be most easily achieved following the recent work on the zero-temperature case [21].

\section{A. Virial theorem in a canonical ensemble}

For this purpose, consider a general Hamiltonian $H=$ $K+I+U$ that describes $N$ particles with arbitrary statistics in arbitrary dimensions, where $K$ is the kinetic energy, $I$ is the interaction, and $U$ is an arbitrary external potential. For ultracold quantum gases, the external potential is simply $U=\sum_{i=1}^{N} U_{i}\left(\mathbf{r}_{\mathbf{i}}\right)$, where $U_{i}\left(\mathbf{r}_{\mathbf{i}}\right)$ has approximately harmonic dependence on the position $\mathbf{r}_{\mathbf{i}}$ of the particles.

In general, via a dimensional analysis, $U$ can be written as $U\left(\mathbf{r}_{1}, \ldots, \mathbf{r}_{\mathbf{N}}\right)=\left(\hbar^{2} \lambda^{2} / M\right) u\left(\lambda \mathbf{r}_{1}, \ldots, \lambda \mathbf{r}_{\mathbf{N}}\right)$, where $\lambda$ has the dimension of the inverse of a length and $u$ is a dimensionless function of its parameters. Therefore, we can use the IHFT given in Eq. (3) to obtain $\lambda \partial E / \partial \lambda=4(1-T \partial / \partial T) E_{t r}$, where $E_{t r}$ is the effective trapping energy defined in Eq. (15). In addition, we can write the energy $E$, again via a dimensional analysis, as $E\left(\ell_{1}, \ldots, \ell_{p}\right)=\left(\hbar^{2} \lambda^{2} / M\right) e\left(\lambda \ell_{1}, \ldots, \lambda \ell_{p}\right)$, where $\ell_{q}$ labels $p$ parameters (all with the dimension of a length) that $E$ may depend on for a given $H$ and $e$ is a dimensionless function of its parameters. Evaluating the derivative with respect to $\lambda$ for fixed values of $\ell_{q}$, we obtain $\lambda \partial E / \partial \lambda=$ $2 E+\sum_{q=1}^{p} \ell_{q} \partial E / \partial \ell_{q}$. Then, the virial theorem is obtained by combining these two analyses, leading to

$$
E=2\left(1-T \frac{\partial}{\partial T}\right) E_{t r}-\frac{1}{2} \sum_{q=1}^{p} \ell_{q} \frac{\partial E}{\partial \ell_{q}} .
$$

Compared to the zero-temperature expression [21], the main differences here are an extra $T \partial / \partial T$ term in front of the potential terms and an extra $\ell_{q}$ term associated with the temperature.

\section{B. Trapped quantum gases}

In particular, for the ultracold quantum gases, which are well described by the $s$-wave scattering length $a$, Eq. (27) reduces to $(1-T \partial / \partial T)\left(E-2 E_{t r}\right)=-(a / 2) \partial E / \partial a$. Note that for finite $k_{c}$, i.e., nonzero interaction range, there would be an additional $-\left(k_{c} / 2\right) \partial E / \partial k_{c}=-\hbar^{2} C k_{c} /\left(4 \pi^{2} M\right)$ term on the right-hand side of this equation. Furthermore, using the IST for the average energy given in Eq. (10) for the last term, the solution of the resultant differential equation [28] can be written as

$$
E=2 E_{t r}-\frac{\hbar^{2} C}{8 \pi M a}+\kappa T
$$


where $\kappa$ is a real constant independent of $T$. This is the most general form of the virial theorem in a canonical ensemble. Compared to the zero-temperature expression [4,5,21], the main difference here is an extra $\kappa T$ term. In the unitarity $a \rightarrow \pm \infty$ limit, it was shown via a dimensional analysis that the virial theorem in a canonical ensemble does not have the last term [24], and hence, we know that $\kappa$ vanishes in this limit, i.e., $\kappa_{a \rightarrow \pm \infty}=0$. We suspect $\kappa=0$ for all $a$; however, since a nonzero $\kappa$ is allowed in general, this possibility needs to be clarified via other means.

Similar to the dimensional analysis that led to Eq. (20), it can be shown that Eq. (28) follows from a dimensional analysis of the average energy $E$ supplied with Eq. (10). Note that $E$ is a function of $T, N_{\sigma}, a$, and the trapping frequency $\omega$ for a trapped system. However, applying a dimensional analysis to the free energy $F$ of trapped systems, which is also a function of $T, N_{\sigma}, a$, and $\omega$, and using Eq. (9), we obtain Eq. (28) with $\kappa=0$ [29]. Therefore, we conclude that $\kappa=0$ for all parameter space, i.e.,

$$
E=2 E_{t r}-\frac{\hbar^{2} C}{8 \pi M a},
$$

and that the virial theorem in a canonical ensemble is of the same form as the zero-temperature one $[4,5,21]$. We recall that taking the derivative of Eq. (29) with respect to $a$ and using the IST for the energy given in Eq. (10), we obtained the IST for the trapping energy given in Eq. (14).

\section{CONCLUSIONS}

To conclude, first we derived the isothermal HellmannFeynman theorem that is suitable for mixed states in a canonical ensemble. Then, we obtained the isothermal magnetic-field sweep theorems for the free, average, and trapping energies and for the entropy, specific heat, pressure, and atomic compressibility of strongly correlated ultracold quantum gases. We applied the sweep theorems to two-component Fermi gases in the weakly interacting BCS and BEC limits and showed that the temperature dependence of the contact parameter could be determined by varying either the entropy or specific heat with respect to the scattering length. We also obtained the virial theorem in a canonical ensemble and discussed its implications for quantum gases.

One of the major challenges for experiments with ultracold quantum gases is the lack of a precise thermometry, and even the measure of the temperature itself for strongly interacting Fermi gases is a challenging problem [30]. On one hand, this makes it more difficult to perform an isothermal (constant temperature) magnetic-field sweep at ultracold temperatures, compared to an adiabatic (constant entropy) sweep that is routinely performed in atomic systems, while tuning the scattering length. On the other hand, it is theoretically easier to calculate thermodynamic quantities at constant temperature; e.g., the calculation of isothermal atomic compressibility is much easier than the isoentropic atomic compressibility in the BCS-BEC crossover. Therefore, the isothermal HellmannFeynman and sweep theorems that we discussed in this paper are probably most useful for other theoretical or numerical studies and possibly for some special experiments where the thermometry is not an issue.

\section{ACKNOWLEDGMENTS}

The author thanks Eric Braaten and Felix Werner for comments. This work is financially supported by the Marie Curie International Reintegration (Grant No. FP7-PEOPLEIRG-2010-268239) and the Scientific and Technological Research Council of Turkey (Career Grant No. TÜBİTAK-3501110T839).
[1] See the recent reviews by F. Werner and Y. Castin, e-print arXiv:1001.0774; and also by E. Braaten, e-print arXiv:1008.2922.

[2] S. Tan, Ann. Phys. 323, 2952 (2008).

[3] S. Tan, Ann. Phys. 323, 2971 (2008).

[4] S. Tan, Ann. Phys. 323, 2987 (2008).

[5] E. Braaten and L. Platter, Phys. Rev. Lett. 100, 205301 (2008).

[6] E. Braaten, D. Kang, and L. Platter, Phys. Rev. A 78, 053606 (2008).

[7] S. Zhang and A. J. Leggett, Phys. Rev. A 79, 023601 (2009).

[8] R. Combescot, F. Alzetto, and X. Leyronas, Phys. Rev. A 79, 053640 (2009).

[9] F. Werner, L. Tarruell, and Y. Castin, Eur. Phys. J. B 68, 401 (2009).

[10] D. Blume and K. M. Daily, Phys. Rev. A 80, 053626 (2009).

[11] G. Baym, C. J. Pethick, Z. Yu, and M. W. Zwierlein, Phys. Rev. Lett. 99, 190407 (2007).

[12] R. Haussmann, M. Punk, and W. Zwerger, Phys. Rev. A 80, 063612 (2009).

[13] H. Hu, X.-J. Liu, and P. D. Drummond, Europhys. Lett. 91, 20005 (2010).
[14] D. T. Son and E. G. Thompson, Phys. Rev. A 81, 063634 (2010).

[15] E. Taylor and M. Randeria, Phys. Rev. A 81, 053610 (2010).

[16] W. Schneider and M. Randeria, Phys. Rev. A 81, 021601(R) (2010).

[17] J. T. Stewart, J. P. Gaebler, T. E. Drake, and D. S. Jin, Phys. Rev. Lett. 104, 235301 (2010).

[18] E. D. Kuhnle, H. Hu, X.-J. Liu, P. Dyke, M. Mark, P. D. Drummond, P. Hannaford, and C. J. Vale, Phys. Rev. Lett. 105, 070402 (2010).

[19] Z. Yu, G. M. Bruun, and G. Baym, Phys. Rev. A 80, 023615 (2009).

[20] F. Palestini, A. Perali, P. Pieri, and G. C. Strinati, Phys. Rev. A 82, 021605(R) (2010).

[21] F. Werner, Phys. Rev. A 78, 025601 (2008).

[22] D. T. Son, e-print arXiv:0707.1851.

[23] J. E. Thomas, Phys. Rev. A 78, 013630 (2008).

[24] J. E. Thomas, J. Kinast, and A. Turlapov, Phys. Rev. Lett. 95, 120402 (2005).

[25] M. Iskin and C. A. R. Sá de Melo, Phys. Rev. B 72, 224513 (2005); Phys. Rev. A 74, 013608 (2006).

[26] C. Sanner, E. J. Su, A. Keshet, R. Gommers, Y. I. Shin, W. Huang, and W. Ketterle, Phys. Rev. Lett. 105, 040402 (2010). 
[27] T. Müller, B. Zimmermann, J. Meineke, J.-P. Brantut, T. Esslinger, and H. Moritz, Phys. Rev. Lett. 105, 040401 (2010).

[28] After the substitution, the resultant equation is of the form (1 $T \partial / \partial T) y=0$, for which the general solution can be written as $y=\kappa T$, where $\kappa$ is a real constant independent of $T$.

[29] This was pointed out to us by E. Braaten (private communication).

[30] So far, there are two model-independent thermometry methods employed in the experiments. The first one is used in
M. Zwierlein et al. [Nature 442, 54 (2006)] for imbalanced Fermi gases, and it is based on fitting the noninteracting edge of the majority component to the Fermi function. The second one is used in L. Luo, B. Clancy, J. Joseph, J. Kinast, and J. E. Thomas [Phys. Rev. Lett. 98, 080402 (2007)], where the temperature is obtained from the thermodynamic relation $T=\partial E / \partial S$, after measuring the energy and entropy to obtain a smooth $E(S)$ curve. The latter method is applicable to both balanced and imbalanced Fermi gases. 